\title{
resources
}

ISSN 2079-9276

www.mdpi.com/journal/resources

Editorial

\section{Acknowledgement to Reviewers of Resources in 2014}

Resources Editorial Office, MDPI AG, Klybeckstrasse 64, CH-4057 Basel, Switzerland

Published: 8 January 2015

The editors of Resources would like to express their sincere gratitude to the following reviewers for assessing manuscripts in 2014:

\begin{tabular}{|c|c|c|}
\hline Ailliot, Pierre & Eggert, Roderick G. & Lee, In \\
\hline Albadi, M. H. & Fertig, Emily & Leitch, Anne M. \\
\hline Allenby, Braden & Flaherty, Eoin & Lepawsky, Josh \\
\hline Atwood, David A. & Foley, Aoife & Lockwood, Michael \\
\hline Axaopoulos, Petros & Fray, Derek J. & Louie, Henry \\
\hline Bak-Jensen, Birgitte & Friedman, Cynthia M. Ross & Lüdeke-Freund, Florian \\
\hline Beckmann, Volker & Funder, Mikkel & Manicad, Gigi \\
\hline Behrens, Arno & Gaeta, Davide & Markard, Jochen \\
\hline Bett, Philip & Giljum, Stefan & Medellin-Azuara, Josue \\
\hline Blonbou, Ruddy & Goldstein, Wendy & Michelsen, Ari M. \\
\hline Bucolo, Sam & Graedel, Thomas & Monz, Christopher \\
\hline Busch, Jonathan & Grothe, Oliver & Morgera, Elisa \\
\hline Bussey, Marcus & Gyning, Sven-Erik & Morse, Wayde C. \\
\hline Calif, Rudy & He, Ping & Mueller, Adrian \\
\hline Carling, Greg & Helmrich, Robert & Murakami, Shinsuke \\
\hline Carta, José Antonio & Hilson, Gavin & Nawri, Nikolai \\
\hline Chang, Tian Pau & Holttinen, Hannele & Niccolucci, Valentina \\
\hline Crawford, Terry L. & Hoornweg, Daniel & Niggli, Urs \\
\hline De La Rosa, Juan José González & Isozaki, Hiroji & Niza, Samuel \\
\hline Del Río, Pablo & Kanemoto, Keiichiro & Patrizi, Nicoletta \\
\hline Deligiorgi, Despina & Kaushal, Priyanka & Pauliuk, Stefan \\
\hline Dempewolf, Hannes & Kent, Jenny & Peinke, Joachim \\
\hline Dijkema, Gerard & Krausmann, Fridolin & Perrotti, Daniela \\
\hline Dyer, Jen & Ladenburg, Jacob & Petroni, Filippo \\
\hline Egbendewe-Mondzozo, Aklesso & Lee, Duehee & Pichler, Paul \\
\hline
\end{tabular}


Prior, Timothy

Pritchard, Geoffrey

Pulselli, Riccardo Maria

Rahimi, Ebrahim

Rankin, John

Reh, Lothar

Rezaie, Behnaz

Roos, Goran

Ryan, Allanah

Sakmar, Susan L.

Santhanam-Martin, Michael
Saurat, Mathieu

Schaffers, Hans

Schmandt, Jurgen

Schnabel, Annette

Semeraro, Teodoro

Smith, Charles W.

Söderholm, Patrik

Strachan, Mark

Subramanian, Suneetha M.

Thomas, Deborah Sk

Thompson, Michael
Van der Voorn, Tom

Veiga, Marcello

Wagner, Jeffrey

Wallsten, Björn

Wang, Haichao

Watanabe, Kazuo N.

West, James

Wilts, Henning

Wilts, Hennning

Wolfert, J.

Young, Steven B.

(C) 2015 by the authors; licensee MDPI, Basel, Switzerland. This article is an open access article distributed under the terms and conditions of the Creative Commons Attribution license (http://creativecommons.org/licenses/by/4.0/). 